\title{
Effects of Light Emissions from LCD Monitors on Public Health
}

\author{
Wilson $\mathrm{Yu}^{1}$, Bobby Sidhu' ${ }^{2}$, Abderrachid Zitouni ${ }^{3}$ \\ 1 Lead Author, School of Health Sciences, British Columbia Institute of Technology, Burnaby, BC \\ 2 Supervisor, School of Health Sciences, British Columbia Institute of Technology, Burnaby, BC \\ 3 Contributor, Environmental Health Services, BC Centre for Disease Control, Vancouver, BC
}

\begin{abstract}
Background: Evidence suggests that exposure to prolonged use of computer monitors may lead to eye discomforts such as eyestrain, blurred vision, headaches, as well as computer vision syndrome. With the increase of internet and computer use at school and work, it raises a serious concern on whether or not the users are affected negatively. Objectives: The following study focuses on estimating the risk of using computers at the British Columbia Technology of Institute (BCIT) laboratories to determine if there is sufficient room lighting (illuminance) and monitor brightness (luminance) to ensure the safety of students at the school.

Methods: Illuminance and luminance were both measured by means of a calibrated Unfors Xi light detector connected to a base unit. Several areas of each environment and monitor were measured and average values were taken to ensure that the data was reliable. The data were compared to applicable standards to determine if room lighting and computer brightness at BCIT were appropriate for safe student use.

Results: The average illuminance was measured to be 405.4 Lux when the recommended level is 500 Lux. The results are considered statistically significant ( $\mathrm{p}$-value $=2 \times 10^{-6}$. On the other hand, the luminance measurements averaged a reading of $94.6 \mathrm{~cd} / \mathrm{cm}^{2}$ when the recommended minimum luminance level is $80 \mathrm{~cd} / \mathrm{cm}^{2}$ (p-value = 0.99964).

Discussion: The collected data show that the illuminance inside the BCIT laboratories where testing was conducted is not sufficient while the luminance of computer screens was found acceptable.

Conclusion: The measurement of the illuminance inside BCIT laboratories showed that room lighting was insufficient and alternations of the monitor's brightness were made to compensate for insufficient lighting. It is recommended to increase room lighting in the tested laboratories in order to have all areas of the laboratory sufficiently lit at all times. This is to ensure that all students at BCIT are provided with a safe and healthy environment for computer use.
\end{abstract}

Keywords: LCD, illuminance, luminance, monitors, Unfors Xi, light

\section{INTRODUCTION}

Whether it is computer monitors, cell phones, TV, tablets, or other electronic devices, liquid crystal display (LCD) screens are viewed each and every day by the public. With the increasing amount of public exposure to these devices, health specialists are becoming concerned with the possible adverse effects associated with long term use.

Light is everywhere, and it is important for society to understand the advantages and disadvantages associated to its exposure each day. Some benefits to light include hazard prevention or eyestrain reduction, but it is mainly used for the ease of viewing objects especially at night (Health and Safety Executive,
1997). Computer monitors, phones, and tablets are all products that emit visible light for the human eye to perceive. While these products are beneficial to our everyday activities, visible light can pose health risks which the public may not be aware of. An example could be something simple like eye dryness or, in some severe cases as shown in several studies discussed later, an increase risk for the development of cancer. Thus, it is important to further these studies to educate not only ourselves but to increase the public's awareness on the effects of light.

The focus of this study will be based on the optical emissions from a variety of LCD computer monitors under different settings and environment. Data will be collected from several laboratories at British Columbia Institute of Technology (BCIT) to 
determine if the environment plays a factor to the light emissions from a computer. The illuminance inside $f$ the rooms and the luminance of the monitors will be measured using a calibrated photometer. Illuminance describes the measure of light falling on a surface and luminance is the amount of light emitted from a surface (Canadian Centre for Occupational Health and Safety [CCOHS], 2013). The collected data will then be analyzed and compared with international standards to determine if there is a health concern regarding light exposure due to long term use of viewing screens.

The researcher is interested in conducting the following study because he is exposed to long term use of computer monitors each day and is concerned with the adverse effects it may cause to his own health as well as the public's. As an individual still in school, the researcher is well aware of the amount of light exposure students are subjected to each day. With society's continuing technological advancement, it is important to understand how we can prevent the harmful effects of light emissions and recommend a safer and more efficient use of computer in a properly illuminated environment.

\section{LITERATURE REVIEW}

\section{Visible Light and its Benefits}

While light does bring its benefits to society in many ways, it is suspected to be associated with few adverse health effects. Some of these effects include anxiety, stress, headaches, and fatigue (Accidental Compensation Corporation [ACC], 2010).

\section{Health Effects Concerned with Exposure to Computer Monitors}

Due to the previous studies mentioned, concern is raised on whether or not light exposure from computer monitors can cause similar health effects. While the experiments conducted in prior studies have shown a relationship between nocturnal light exposure and cancer growth, more research needs to be done regarding the effects of light emitted from computer monitors rather than surrounding light to truly understand the health effects associated with LCD monitors.

According to a document released by the American Optometric Association, individuals who are exposed to long-term use of computers complain about symptoms such as eye discomfort and stress (American Optometric Association [AOA], 1997). An increase of exposure of the eye over time to computer monitors seems to lead to an increase in discomfort. Based on a case study conducted by the University of Benin in Nigeria, participants to the survey who were exposed to a computer monitor for more than 8 hours responded with higher numbers of complaints (35.9\%) which included eyestrain, blurred vision, and headaches compared to individuals who spent only 1-2 hours (17.5\%) (Chiemeke, IAENG, Akhahowa, Ajayi, 2007). These studies revealed sufficient evidence that long term exposure to computer monitors can potentially cause adverse health effects.

It is also known that individuals who are exposed to computer monitors for prolonged periods of time can develop vision related problems identified as computer vision syndrome, or CVS (Bali, Navin, Thakur, 2007). In one study, 300 Indian ophthalmologists were surveyed on the effects of light exposure from computer monitors. According to the researcher, CVS occurs after an individual is exposed to a computer for more than 3.59 hours (Bali et al., 2007). Results from this study indicated that eyestrain is the most common symptom of CVS (97.8\%), with headache being the second highest (82.1\%), and tiredness and burning sensation as the third (79.1\%). Furthermore, the researcher noticed those who used computers for extensive periods had dry eyes due to a decrease in blinking. An individual with dry eyes will not only experience eye discomfort and irritation but also blurring in the vision (Royal College of Ophthalmologist, 2013).

Another study confirmed that eye blinking is certainly reduced in those exposed to computer monitors by analyzing the spontaneous eye link rate (SEBR) of 51 individuals before and during exposure (Freudenthaler, Neuf, Kadner, Schlote, 2003). After the testing, the results showed that blinking during time of exposure was lower (5.34 \pm 4.53 eye blinks/min) when compared to time of unexposed (15.54 \pm 13.74 eye blinks/min). It also shows that exposure to computer monitors for a lengthy period of time will result in dry eyes due to the reduction of blinking.

Based on these studies, it seems probable that a relationship does exist between long term exposure to computer monitors and adverse health effects. A theory may then be generated by utilizing the collected data as a reference and predicting that individuals subjected to prolonged periods of light from LCD monitors have a higher chance of 
experiencing adverse health effects in general.

\section{Legislation, Guidelines, and Recommendations}

According to the study conducted in 2007 at the University of Benin, $85 \%$ of those surveyed indicated that they did not have ergonomics guidelines at their workplace (Chiemeke et al., 2007). Despite their willingness to find a solution to the problem, most operators did not take action to provide their employees with improved practices. In addition, Environmental Health Officers do not have jurisdiction over the issue because of lack of regulations on the matter.

The study at the University of Benin, however, does provide suggestions on how workers can protect themselves if exposed to long term daily use of computers. For example, rooms should always have enough light to ensure clear vision of the screen and illumination in the room should be at least 3 times brighter than the monitors (Chiemeke et al., 2007). Individuals should also increase blinking to reduce dryness of eyes and take short breakson a regular basis. Since glare can cause blurred vision and headaches, an antiglare screen can also be placed in front of the monitor to protect users. Finally, a viewing distance of 20-40 inches at a viewing angle of less than 15 degrees from the computer monitor is suggested (Chiemeke et al., 2007). While these recommendations are not set in stone, they do indicate the importance of healthy computer use and awareness to the issues that need to be addressed.

While most work environments do not have ergonomics guidelines to address the health concerns of their employees, the Department of Labour in New Zealand issued guidelines in November 2010 that provide a basis on how computers should be used to reduce discomfort and pain (ACC, 2010). This document explains the potential health issues associated with long-term use of computers, and how associated hazards can be controlled in a work setting. The guidelines provide a series of recommendations on lighting requirements and other factors such as posture and individual reasons that could affect the individual's wellbeing when using a computer. Although regulatory requirements on computer screen brightness are not always available, , more and more workplaces are showing awareness of the problem and implementing practical measures to prevent or reduce eye discomfort associated with computer use.
While many workplaces do not have ergonomic guidelines related to computer screens, guidelines from WorkSafeBC and Canadian Centre for Occupational Health and Safety do exist (CCOHS, 2013; WorkSafeBC, 2009). For example, CCOHS provides useful information on appropriate illuminance levels for various activities in different work environments. According to their guidelines, individuals who are subjected to a computer for visual tasks of medium contrast or small size will require a light level of 500-1000 lux (CCOHS, 2013). WorkSafeBC also states that 500 lux is needed in order to work safely and efficiently for prolonged periods (WorkSafeBC, 2013). According to WorkSafeBC, the illumination levels stated must be provided and maintained (Section 4.65, Occupational Health and Safety Regulation, 2013). The regulations and guidelines for both WorkSafeBC and CCOHS, however, mainly specify the illumination levels (room lighting) required for the environment but not the luminance levels from computer monitors. Thus, more research needs to be conducted regarding the recommended luminance levels for LCD monitors so that the general public can be given a standard to follow for these specific devices used every day.

\section{Summary}

As humans advance, so will technology. Due to investing half of our time each day to the use of electronic devices, we need to be constantly aware of the possible effects they may cause to our health.. Potential health effects associated with exposure to light emissions from computer monitors are being studied more extensively in our modern society as awareness of such effects keeps gaining ground worldwide. With the invention of the computer and the availability of internet, most organizations today use such technologies to operate their business. Individuals who are subjected to prolonged use of computers each day could find their health adversely affected after few years of use. The rationale for this study is to determine what recommendations can be made for LCD monitors to ensure that users feel comfortable and safe during periods of long exposure.

The purpose of this study is to determine if the measured light emitted from LCD computer monitors in a variety of locations at BCIT and compare the results to the levels recommended in Canada. The study will also help verify if the suggested null hypothesis is true, $\mathrm{H}_{\mathrm{o} \text { (illum) }}=500$ lux and $\mathrm{H}_{\mathrm{o}(\mathrm{lum})}=80$ $\mathrm{cd} / \mathrm{cm}^{2}$, so that LCD monitors and room illuminance at BCIT laboratories will meet international standards. The alternative hypothesis will then be, $\mathrm{H}_{\mathrm{A}(\mathrm{illum})} \neq 500$ lux and $\mathrm{H}_{\mathrm{A}(\mathrm{lum})} \leq 80 \mathrm{~cd} / \mathrm{cm}^{2}$, so that LCD monitors 
and room illuminance at BCIT laboratories do not meet international standards.

Data was collected in this study and analyzed by conducting a statistical analysis. The test was then checked to determine if the suggested hypothesis should be accepted or rejected. The results from the collected data were then compared with international standards to determine if there were potential health issues regarding long term exposure to light from LCD monitors from BCIT laboratories. Recommendations were then constructed based on these findings to ensure safe and efficient use of computer screens.

\section{MATERIALS AND METHODS}

\section{Description of Materials}

This study was conducted using the materials listed above to collect measurements of illuminance inside the rooms and luminance from LCD computer monitors. Unfors Xi Platinum Edition, a calibrated photometer, was used by the researcher to perform the tests. The instrument, which is digital, consists of a base and for a light detector for measuring ambient and emitted light (Unfors, n.d.). The base component (the reader) allows the necessary settings and adjustments prior to measurements. Furthermore, the base can be connected to a computer to record the collected data using the software Unfors Xi View

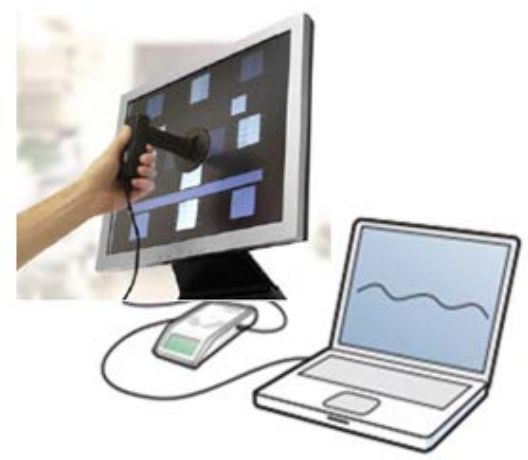

Figure 1. Unfors Xi Platinum Edition Setup
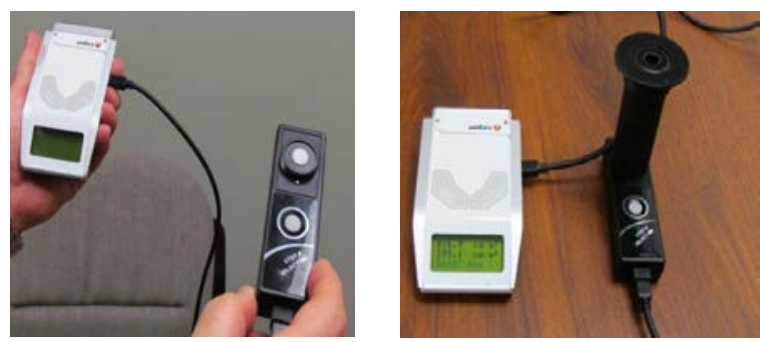

Figure 2. Light Detector without (left) and with (right) luminance tube

\section{Description of Methods}

Data for this study was collected by measuring both the illuminance of the room and the luminance (brightness) of LCD monitors. The only practical difference between the two types of measurement was that luminance required a tube to be mounted onto the light detector prior to measurement of optical emission as seen in figure 2. The purpose of this topic was to measure the brightness of a selection of viewing screens at various laboratories at BCIT under different room lighting conditions. In addition, viewing screens under different settings were selected to determine if there was a difference in the data collected.

To account for the environment, the illuminance was measured by placing the probe 0.5 meters away from the monitor in 3 different areas (left, right, and center) to determine the brightness surrounding the computer screen. The method used for detecting light emission directly from the monitor (luminance) can be seen in figure 3 where 5 locations of the monitor were selected for measurement. The center and each corner of the monitor were measured to obtain the average brightness from the viewing screens. 


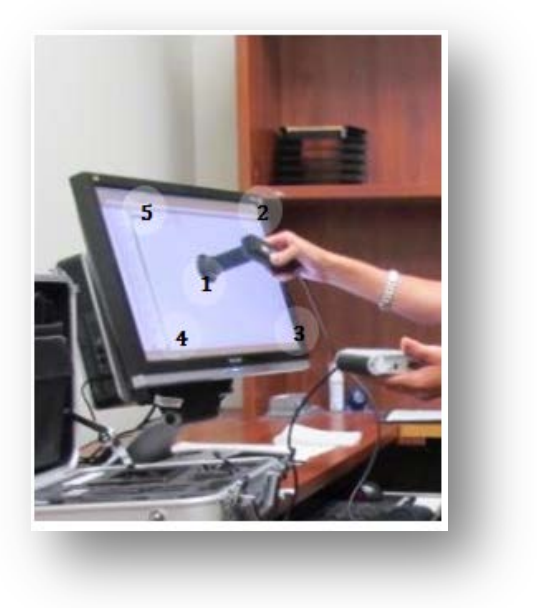

Figure 3. UnforsXi Light Detector Measuring 5 Areas of Monitor

The base component of the Unfors Xi was connected to the computer via the RS-232 cable which allowed the collected data from the light detector to be transferred to the software Unfors Xi View. The information received in the program was then exported to Microsoft Excel for further statistical analysis (Unfors, n.d.). These values were then compared with international standards from Illuminating Engineering Society (IES), which displayed various amounts of required illumination levels for different types of environments (Illuminating Engineering Society [IES], n.d.). Based on the values collected, the researcher made recommendations to the public on how LCD monitors could be used in a more safe and efficient manner.

\section{Reliability and Validity of Measures}

For this study, the reliability would be considered high because the researcher was following a fixed procedure suggested by BCCDC. The instrument had been calibrated and was very accurate in terms of detecting and measuring light emissions. Specific areas of the monitor were repeatedly measured to ensure that the data collected was accurate and reliable. The validity of this experiment should also be considered high because the Unfors $\mathrm{Xi}$ light detector is designed for measuring illuminance of viewing rooms and luminance of viewing monitors (Unfors, n.d.). In addition, only the researcher collected the readings to ensure consistency throughout the study. Therefore, the overall study should be considered respectable due to the methods used to carry out the experiment.

\section{Calibration of Instruments}

Instruments prior to use need to be calibrated to ensure that measurements collected are accurate. If an instrument is not calibrated, then the data could be incorrect and lead to false conclusions. For this study, the Unfors Xi Platinum Edition had been calibrated three months prior to the experiment. According to the Unfors Xi user manual, the light detector is calibrated to international traceable standards (NIST, PTB). Therefore, the data collected with the instrument is considered accurate and reliable when measuring illuminance and luminance.

\section{Inclusion and Exclusion Criteria}

The only LCD monitors that were included in this study were from the BCIT laboratories. Most of the viewing screens in the laboratories were identical in terms of size and brand name, and were representative of what students were exposed to every day for prolonged periods. In addition, monitors under different settings such as brightness were tested for in the laboratory. This allowed the researcher to determine if there was a difference in optical emissions under an altered environment (3 different laboratories). Any computer monitor not from BCIT or in the 3 laboratories being tested were excluded from this study. This includes computers available in the hallway and other public areas at BCIT.

\section{Ethical Considerations}

Ethical considerations were not applicable to this study because it did not require a survey and no humans were involved ( $\mathrm{H}$. Heacock, personal communication, November 5, 2013).

\section{Pilot Study}

The purpose of a pilot study is to ensure that our method of collecting data is reliable and valid prior to the actual experiment (National Centre for the Replacement, Refinement and Reduction of Animals in Research [NC3Rs], 2006). The researcher measured five spots of the monitor 5 times prior to the actual test to determine if the data was consistent. Thus, there is reason to believe that the information gathered yielded a respectable conclusion. The pilot study was conducted on December 17, 2013. 


\begin{tabular}{|l|l|l|l|l|}
\hline & Mean & Median & Mode & Standard Deviation \\
\hline Illuminance & 405.4 & 436.3 & N/A & 70.4 \\
\hline Luminance & 94.6 & 95.8 & N/A & 21.1 \\
\hline
\end{tabular}

Table 1. Descriptive Statistics for Illuminance and Luminance Collected Data.

\section{STATISTICAL ANALYSIS}

\section{Descriptive Statistics and Data Type}

In this study, numerical discrete data was used as it describes any quantity defined by a number (Heacock \& Sidhu, 2013). Illuminance and luminance levels were measured in this experiment in units of Lux and $\mathrm{cd} / \mathrm{cm}^{2}$, respectively. As stated previously, five spots on the monitor were measured to determine the average optical emissions (luminance) from the monitor. In addition, several areas around the monitor were measured to determine the average illuminance (room lighting) in the room. The mean values were calculated and compared with international standards to determine if the values were higher or lower than the stated regulations as seen in Table 1. The median and mode were also evaluated, as well as the standard deviation to determine the possible variation from the determined mean value. Furthermore, the range of the data was analyzed to seek outliers. Additional graphs of the average illuminance and luminance can be seen in Figure 4 and 5.

\section{Inferential Statistics}

Inferential statistics is used to conclude from the sample to the population (Heacock \& Sidhu, 2013). In other words, it is used to form conclusions with the use of statistics to determine if the generated hypothesis is true. For this study, a one sample t-test was used to determine if the values measured were equal to the null hypothesis (the international standard value). If the values were greater, then there should be an excessive amount of light exposure and health effects may be of concern. If the values were significantly lower than the null hypothesis, then there is not enough light to meet the required standard to ensure comfortable work for the public.

The p-value was set to 0.05 which means there was a $5 \%$ chance that the results could be insignificant and be due to chance.

\section{Statistical Package}

For the purpose of this study, NCSS 9 was used for statistical analysis on the data collected with the Unfors Xi light detector (Hintze, 2013). Microsoft Excel was also utilized to compile the data collected from the software Unfors $\mathrm{Xi}$ View prior to being transferred to NCSS 9 for further statistical analysis and interpretation (Microsoft, 2007).

\section{RESULTS}

\section{Interpretation of Statistical Results}

In this study, the Ho(illum) $=500$ Lux and Ho(lum) $=$ $80 \mathrm{~cd} / \mathrm{cm}^{2}$. The HA(illum) $\neq 500$ Lux and HA(lum) $\leq 80$. Therefore, 500 Lux is considered the required amount of lighting in a room to ensure that the public is exposed to safe and healthy monitor use. Moreover, the monitor must provide a luminance of $80 \mathrm{~cd} / \mathrm{cm}^{2}$ to ensure users adequate brightness. A total of 240 readings was taken for illuminance and luminance as 5 points of each monitor and 3 specific surrounding areas were measured for each monitor (Total 30 monitors). Averages were then calculated to yield 30 results for each illuminance and luminance. The mean, median, mode, and standard deviation were then calculated with Microsoft Excel and analyzed.

The results can then be further analyzed by looking at the data output by NCSS 9. According to the information collected for illuminance, two of the test assumptions rejected normality and therefore the nonparametric Wilcoxon signed-rank test is used to determine if the results are significant. By looking at the probability level, $2 \times 10^{-6}$ is much lower than 0.05 which means that the data is statistically significantly different from the standard and the null hypothesis should be rejected. Thus, it can be concluded that the illuminance levels are not high enough to meet the required standard (500 lux) as the calculated mean is only 405.4 lux. Therefore, there are possible health concerns associated with insufficient room lighting and recommendations would need to be made to increase the overall brightness of the laboratory. 


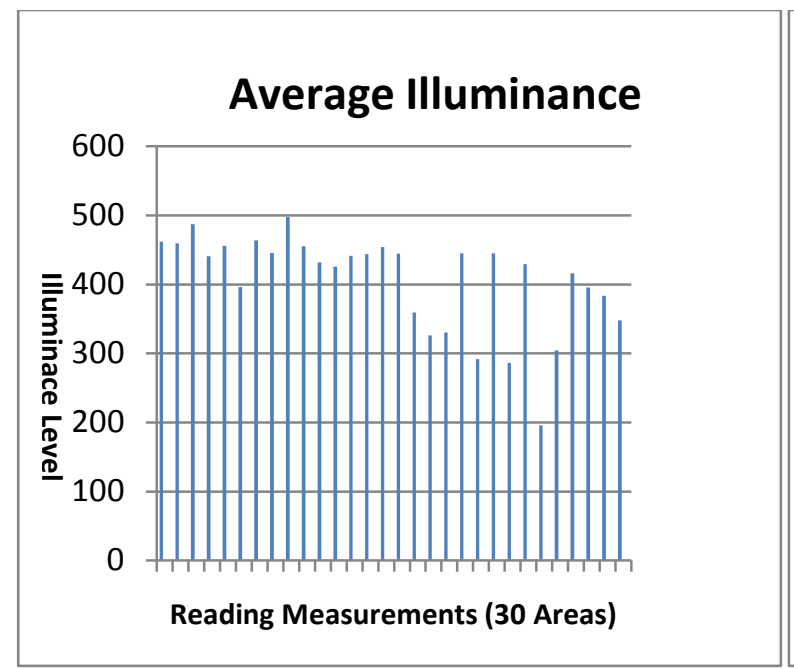

Figure 4. Average illuminance Measurements at BCIT Laboratories 2665, 2625, 2635) for all 30 readings.

Luminance, on the other hand, generated much different results and which led to a different conclusion. The test assumptions could not reject normality and therefore the parametric one-sample ttest is used to determine if the results are significant. By looking at the probability level, 0.99964 is much higher than 0.05 which means that the data is not statistically significant and the null hypothesis should not be rejected. Thus, it can be concluded that the luminance levels are sufficient in the laboratory as the calculated mean value was $94.6 \mathrm{~cd} / \mathrm{cm}^{2}$. Therefore, health concerns associated with inadequate monitor brightness may not be a significant issue at BCIT.

\section{Alpha and Beta Errors}

For this study, the null hypothesis for the illuminance test was rejected. Thus, it is possible that a Type I error may occur but is unlikely because of the p-value $2 \times 10^{-6}$. If the alpha value was changed from 0.05 to 0.01 , the p-value would still be lower. Thus, the chance that a type I error would occur is considered extremely low. For the luminance test, the null hypothesis was not rejected. This means that a Type II error could possibly have occurred but is unlikely due to the high p-value of 0.99964 .

Since the null hypothesis was rejected for the illuminance test, the power of the study needs to be analyzed to ensure confidence in the rejection. The power is 1.00 and 0.99 when the alpha value is 0.05 and 0.01 respectively. With such a high power, it reinforces that the null hypothesis was rejected correctly and that the results are reliable.

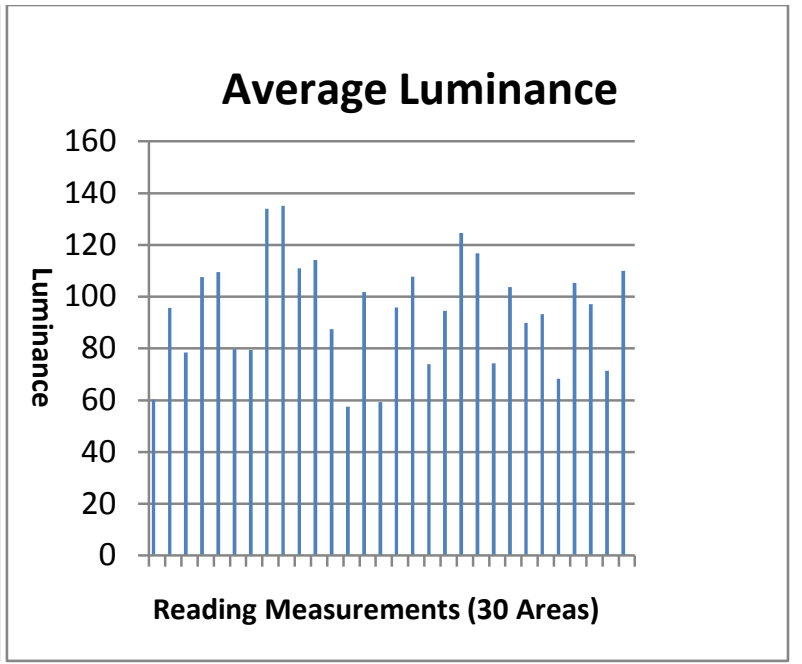

Figure 5. Average luminance Measurements at BCIT Laboratories (SW3 (SW3 2665, 2625, 2635) for all 30 readings.

\section{DISCUSSION}

The results of the survey show evidence that BCIT laboratories in rooms 2665, 2625, and 2635 did not have enough ambient lighting for the users. A minimum lighting of 500 Lux is generally recommended by international organisations to ensure proper lighting of laboratory rooms (Abderrachid Zitouni, personal communication, December 4, 2013). However, this was not the case in the laboratories that were tested in this study as the illuminance in the rooms seemed rather $\mathrm{dim}$ at an average level of 405.4 Lux, significantly below 500 Lux. In addition, the illuminance in the rooms varied from a position to another because of the non uniformity of the lighting from the ceiling. Certain areas are not provided with interior lighting and this explains why some monitors are located in lower illuminance conditions. This is a common problem at the sides of the room or at the back. Areas with light directly above the computer stations also did not provide sufficient amount of illuminance as well. This raises concern on whether or not individuals who use the computers in the laboratory are able to see clearly which could be harmful if exposed for prolonged periods. For example, if users have difficulties to interpret on the contents of the screen, they would tend to focus harder on the screen. This will result in a reduction of blinking which would lead to eye dryness and possibly irritation.

Once the measurement of illumination in the room was conducted, the brightness of the screens was checked as well. Most of the collected readings vary because the brightness was not the same on all monitors. Monitors vary in brightness, and light 
emission is not uniform across viewing screens. This is why the average of 5 areas of the monitor was measured to ensure an accurate and representative estimate of luminance for each station. According to the standards applicable to viewing screens, the required amount of luminance that warrants adequate lighting is must at least $80 \mathrm{~cd} / \mathrm{cm}^{2}$. The collected results show that monitors in the three BCIT laboratories were definitely bright enough with an average luminance of $94.6 \mathrm{~cd} / \mathrm{cm}^{2}$. Thus, the luminance of the monitors meet the applicable standards. One possible reason explaining the higher than needed luminance of the monitors could be the need to compensate for insufficient lighting in the room.

As stated in the literature review, long term exposure to computer monitors will lead to an increase in eye discomfort such as eye strain, blurred vision and headaches. Inadequate lighting of a room in the laboratories may require students to focus more while viewing material on a screen and that could lead to few undesirable problems such as eye dryness and irritation. Therefore, room lighting inside BCIT laboratories should be corrected to ensure that room illuminance meets the recommended standard of 500 lux.

\section{Limitations}

Experiments are never perfect, and this study has several possible uncertainties that should be discussed. Human error always exists in experiments and the researcher himself could make mistakes while conducting the experiment. For example, measuring the luminance of the monitor may not always be in the exact location. Although there is a template of where to place the light detector, the area is not always at the same location. In addition, students moving around in the lab may affect the readings of the light detector as room lighting may be shielded partially. It is unlikely the instrument could have been the source of any error as it was calibrated recently and functioned properly throughout the tests.

Another aspect of the experiment is that only LCD monitors were tested in this study. Light emitting diode (LED) monitors were not available in the laboratories and were, therefore, not used in the experiment. Due to the different lighting LED monitors use, the brightness of the monitors may be more or less bright in general. Also, the researcher had to rely on only one light-measuring device , the Unfors instrument; an additional device would have allowed a comparison of readings. Finally, no survey was conducted with BCIT students regarding the number of hours they spent at the laboratories due to time constraint.

\section{Recommendations}

Recommendations for BCIT would be to increase the amount of lighting in the room in order to meet the requirements applicable to laboratory lighting. The school could also provide lighting in the back of the rooms at all times of the day. This is because the computer stations at the back of the rooms, near the windows, never have light turned on due to the sunlight provided from outside. In the evening, however, the laboratories are still available for use but with less sunlight. With less light available, users tend to increase the monitor brightness to accommodate accordingly.

\section{Future Studies}

Future considerations for this study may include LED monitors. Due to time constraints and the fact that BCIT laboratories consisted only of LCD monitors at the time, the study was limited to one type of monitor. In addition, data could be collected during times when the lab is busy to determine the general illuminance that is representative of a more practical situation. An additional light-measuring instrument could also be used to conduct the study to further validate the data obtained through this study. Finally, a survey should be conducted with BCIT students to determine how much time they spend on average inside the laboratories.

\section{CONCLUSION}

Based on the overall study, BCIT laboratories did not have enough room illumination for computer users. However, it is possible that alteration to the brightness of the monitors have been made to accommodate for insufficient lighting. This can be seen based on the overall brightness of the monitors collected in the study. The increase of brightness of the LCD monitors allows individuals to view more clearly the screens, thus avoiding somehow squinting and focusing excessively. Overall, it is recommended that BCIT increase the brightness of their laboratory rooms as well as have the lights turned on at the back of the room at all times when it is no longer considered bright outside. This will help students at the school do their work in an adequate environment with proper room lighting screen brightness. 


\section{ACKNOWLEDGEMENTS}

The author would like to take this opportunity to thank Abderrachid Zitouni, Provincial Radiation Specialist, from BCCDC for his continued support and guidance throughout the research. This study would not be possible without his expert advice. The author would also like to thank Bobby Sidhu for his assistance and direction with the research paper.

\section{COMPETING INTEREST}

The authors declare that they have no competing interest at this time.

\section{REFERENCES}

Accidental Compensation Corporation [ACC]. (2010). Guidelines for using computers. Department of Labour, 8-18. Retrieved from http://www.acc.co.nz/PRD_EXT_CSMP/groups/ext ernal_ip/documents/guide/wpc090196.pdf

American Optometric Association [AOA]. (1997). The effects of computer use on eye health and vision. Retrieved from http://www.aoa.org/Documents/optometrists/effects -of-computer-use.pdf

Bali, J., Navin, D., \& Thakur, B. R. (2007). Computer vision syndrome: A study of the knowledge, attitudes and practices in Indian Ophthalmologists. Indian Journal of Ophthalmology, 55(4), 289-294.

Canadian Centre for Occupational Health and Safety [CCOHS]. (2013). Lighting ergonomics - survey and solutions. Retrieved from http://www.ccohs.ca/oshanswers/ergonomics/lighti ng_survey.html

Chiemeke, S. C., IAENG, Akhahowa, A. E., \& Ajayi, O. B. (2007). Evaluation of vision-related problems amongst computer users: a case study of university of Benin, Nigeria. Proceedings of the World Congress on Engineering, 1, 217-221.

Electric Lighting History. (n.d.). Retrieved from http://www.iar.unicamp.br/lab/luz/ld/Hist\%F3ria/El ectric\%20Lighting\%20History.pdf
Freudenthaler, N., Neuf, H., Kadner, G., \& Schlote, T. (2003). Characteristics of spontaneous eyeblink activity during video display terminal use in healthy volunteers. Graefe's Archive for Clinical and Experimental Ophthalmology, 241(11), 914-920.

Health and Safety Executive. (1997). Lighting at work. Retrieved from:

http://www.liv.ac.uk/media/livacuk/safety/hazardat oz/lighting_at_work.pdf

Hintze, J. (2013). NCSS 9. NCSS, LLC. Kaysville, Utah, USA. www.ncss.com

Illuminating Engineering Society [IES]. (n.d.). Room illumination level. Retrieved from BCCDC Microsoft. (2007). Microsoft Excel (Version 2007). Microsoft Corporation.

National Centre for the Replacement, Refinement and Reduction of Animals in Research [NC3Rs]. (2006). Why do a pilot study. Retrieved from http://www.nc3rs.org.uk/downloaddoc.asp?id=400

Occupational Health and Safety Regulation [OHSR], B.C. Reg. 404/2012 (2013). Retrieved from http://www.bclaws.ca/EPLibraries/bclaws_new/doc ument/ID/freeside/296_97_00

Poynton, C.A. (1996). Reducing eyestrain from video and computer monitors. Retrieved from http://www.poynton.com/PDFs/Reducing_eyestrain .pdf

Royal College of Ophthalmologists. (2013). Dry eye. Retrieved from

http://www.rnib.org.uk/eyehealth/eyeconditions/eye conditionsdn/Pages/dry_eye.aspx

Unfors. (2011). Xi Platinum Edition User's Manual.

WorkSafeBC. (2009). How to make your computer station fit you. Retrieved from http://www.worksafebc.com/publications/health_an d_safety/by_topic/assets/pdf/comptr_wrkstn.pdf

WorkSafeBC. (2013). Illumination. Retrieved from http://www2.worksafebc.com/Publications/OHSRe gulation/Part4.asp?ReportID=18005 